\title{
Knowledge Description Model for Bodies of Knowledge in Software Engineering Context
}

\author{
Pablo Alejandro Quezada- Sarmiento 123 \\ ${ }^{1}$ Universidad Internacional del Ecuador, Escuela de \\ Informática y Multimedia \\ ${ }^{2}$ Universidad Tecnica Particular de Loja, Department of \\ Electronics and Computer Science; \\ ${ }^{3}$ Universidad Politécnica de Madrid, Departamento de \\ Sistemas Informáticos CITSEM \\ Loja, Quito - Ecuador, Spain - Madrid \\ paquezadasa@internacional.edu.ec,paquezada@utpl.edu.ec \\ Hironori Washizaki \\ Waseda University, Dept. Computer Science and \\ Engineering \\ Tokyo -Japon \\ washizaki@waseda.jp
}

\author{
Juan Garbajosa \\ Universidad Politécnica de Madrid, Departamento de \\ Sistemas Informáticos CITSEM \\ Spain - Madrid \\ jgs@etsisi.upm.es \\ Liliana Enciso \\ Universidad Tecnica Particular de Loja, Department of \\ Electronics and Computer Science \\ Loja- Ecuador \\ lenciso@utpl.edu.ec
}

\begin{abstract}
Bodies of Knowledge (BOK) contains the relevant knowledge for a discipline. BOK must embody the consensus reached by the community for which this BOK will be of application. This consensus is a prerequisite for the adoption of the BOK by the community. In this paper, we utilize a combinations of Software Engineering Body of Knowledge (SWEBOK), models representation, and design science methodology in order to describe the software engineering knowledge context (SEC).

SWEBOK serves as backbone taxonomy, while models representation provides a context of representation. In the process of develop of this paper science design methodology was used to provide fundamental knowledge in software engineering (SE).
\end{abstract}

Keywords - Body of Knowledge, Component, Engineering, Models, Software, Structure Stakeholders, Taxonomies

\section{INTRODUCCIÓN}

A BOK is a term used to represent the complete set of concepts, terms, and activities that make up a professional domain. It encompasses the core teachings, skills and research in a field or industry [1].

A BOK may include technical terms, and theoretical concepts as well as recommended practices [2].

A profession's BOK is its common intellectual ground it is shared by everyone in the profession regardless of employment or engineering discipline [3].

The development of a BOK is a complex activity. It has done through the use of guide knowledge, determining that knowledge can be often represented through Knowledge Areas (KA), which has not met the expectations of stakeholders in BOK's, this is so since BOK are quite extensive and it is not only used by engineering stakeholders but also, for instance, in government [4].

However, it does not yet exist a widely agreed guide- lines for the description of BOK in general, or specifically, for describing the BOK knowledge, a first step towards the development of the BOK. The description the knowledge allows us to understand the context of each discipline, the relation with others disciplines, the structure, contents, and the capacities necessaries for correct develop of BOK [3]. To cover the gap of the description of the BOK knowledge, this paper presents a model to describe a Knowledge in SWEBOK context supported by conceptual models, science design as methodology.

This paper has been structured as follows: Section I, contains the Introduction, section II introduces a Background of BOK, Section III Research Methodology and proposal model. Then Section IV introduces some conclusions, Section V Future Work (The following steps in this research), Sections VI Acknowledgements, and the last section the references that support this research is presented.

\section{BACKGROUND}

A BOK is a collection of substantial concepts, and skills that represent knowledge of a certain area in engineering or scientific discipline, and ensures its common understanding. A BOK may include technical terms and theoretical concepts as well as recommended practices [2]. A BOK is not just a professional reading list, a library, a website or a collection of websites, a description of professional functions or a collection of information. A BOK is a list of knowledge, skills, and abilities (competencies), organized into an integrated structure (taxonomy) with a specific level of accomplishment specified for each competency (proficiency). It is the sum of knowledge 
within a profession that includes proven traditional practices which are widely accepted, emerging innovative practices as well as published and unpublished material. It is a living body of information that requires updating and feeding to remain current [5]. Professional communities have created and used BOK to consolidate their discipline, standardize practices, improve processes, and warehouse community knowledge [2].

$\mathrm{BOK}$ are a relatively recent development in information modeling, but they draw on a rich heritage from other models.

The logical evolution of a BOK in four phases: Controlled Vocabulary [6], Taxonomies [7], Ontology [8], Metamodel [9] [10]. In the case of SE, the best ways to describe the knowledge based in BOK is the use of level to describe KA, Units and Topics with the respective breakdown.

\section{RESEARCH METHODOLOGY}

In order to support this paper, we used a Design Science Research Methodology that is of importance in a discipline oriented to the creation of successful artifacts. The design science research methodology (DSRM) presented here incorporates principles, practices, and procedures required to carry out such research and meets three objectives: it is consistent with prior literature, it provides a nominal process model for doing DS research, and it provides a mental model for presenting and evaluating DS research in this case in the context of SE [11].

We propose and develop a design Science research methodology (DSRM) for the production and presentation of DS research in the description of model for $\mathrm{BOK}$ in the context of SE.

Case: Knowledge Description Model for Bodies of Knowledge in $S E$.

- Activity 1. Problem identification and motivation. - In this activity we defined the specific research problem and justify the value of a solution. The research problem is that it has not a model to describe knowledge in the SEC for this reason we propose a conceptual model based in the analysis of concepts in software engineering context. This solution describes the general structure and related concept of BOK; this model is an artifact to describe the knowledge. This model is important because permit understand the real context of Bok specially the related concepts.

- Activity 2: Define the objectives for a solution. The objectives of this solution were:

Develop a Model to describe knowledge in the SEC.

Analysis of concepts based in SWEBOK that permitted extracted the model.

- Activity 3: Design and development. In this activity we developed the artifact in this case the model to describe knowledge base in the concepts of SWEBOK.

- Activity 4: Demonstration. - The use of the artifact solve one more instances of the problem of Knowledge representation. The artifact permits describe the knowledge in SEC.

- Activity 5: Evaluation. - The artifact supports a solution to the problem; we compared the objectives of the solution to actual observed results from use of artifact demonstration.

- Activity 6: Communication. - Manuscripts relating to the SWEBOK have been published in academic conference proceedings, and professional outlets. Figure 1 summarizes how the DSRM applies to the steps undertaken as part of this DS research effort in the context of this model.

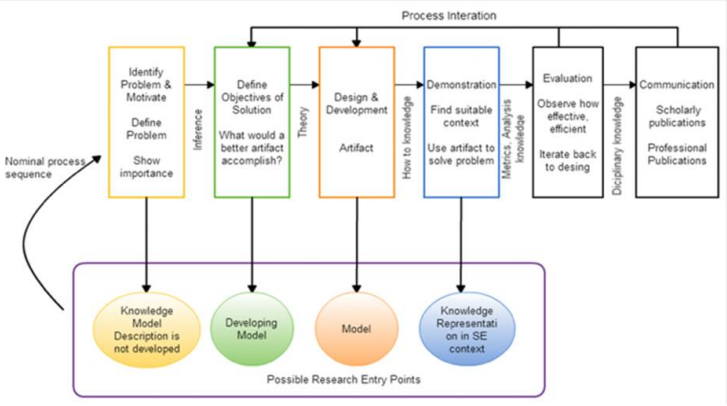

Figure 1. Process of DS in BOK context

Proposal Model: According to [12], new information technologies from text extraction, to data visualization and semantic technologies, introduce a knowledge representation that reflects the view of the actors building the tools of the knowledge they are trying to represent. An underlying knowledge representation reflected in this shift is that scientific discourse is viewed as a purposeful, persuasive text, which is created by a human actor, who aims to influence and persuade human readers.

In the same context [13] presents a general cognitive model for skills, and concepts representation, and a proposal for computational implementation. The model is intended to help bridge some of the natural problems that arise in current massive education models, through the adaptation and personalization of learning environments.

In the area of SE [3] a combination of SWEBOK and text categorization to categorize software engineering knowledge it was used. SWEBOK serves as a backbone taxonomy while text categorization provides a collection of algorithms including knowledge representation, feature enrichment and machine learning.

According to [13], fundamental knowledge types in software engineering are carefully analyzed as well as their characteristics. Then, incorporated with SWEBOK, we propose a knowledge categorization methodology as well as its implementing algorithms. In the same context of knowledge representation is necessary consider the ontology model of software engineering is an effective approach for the intercommunion between people from the different domains, the communication and interoperation among agents, and the share and reuse of the software. But the lack of formal analysis 
tools for domain modeling results in taking liberties with conceptualization.

In the context of representation of SWEBOK based in ontologies, Issues and Techniques [14], [15], [16] [17] proposed an initial models and way of representation and interpretation of Knowledge. This paper proposes a model description based in concept abstracted of SWEBOK, and support by domain and SEC.

In the figure 2 the Knowledge Description Model for Bodies of Knowledge in SEC are showed.

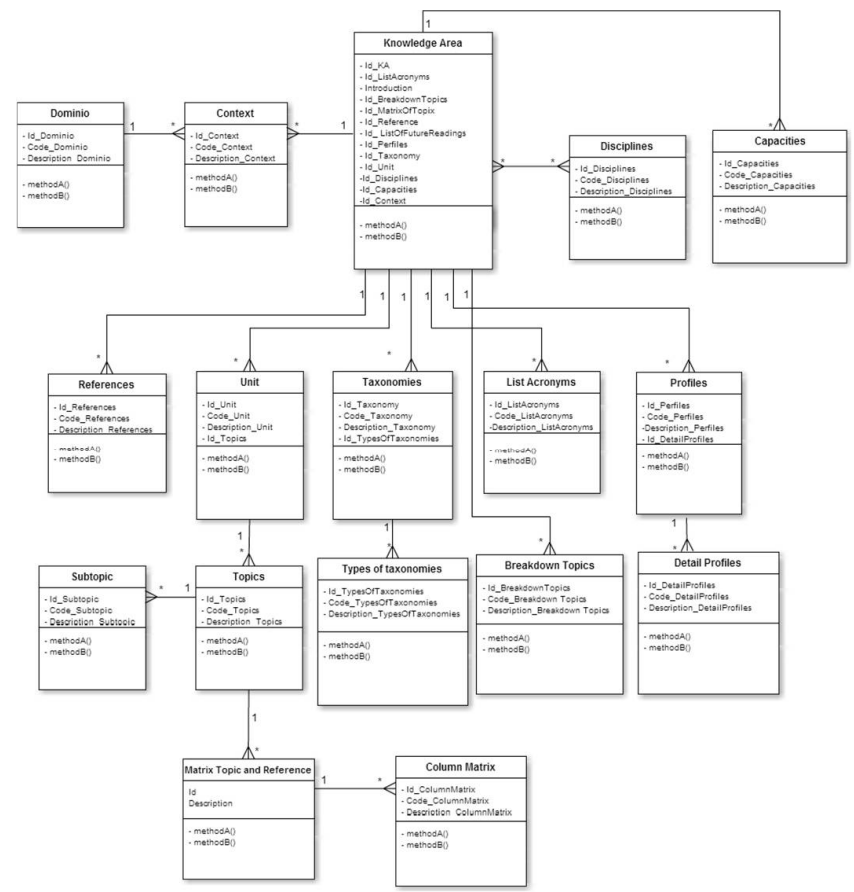

Figure 2: Knowledge Description Model for Bodies of Knowledge in SEC

In this model the primaries concept in SEC is consider in order to develop an ontological model that support knowledge in BOK. In the figure 3 the initial ontology in SE context is showed.

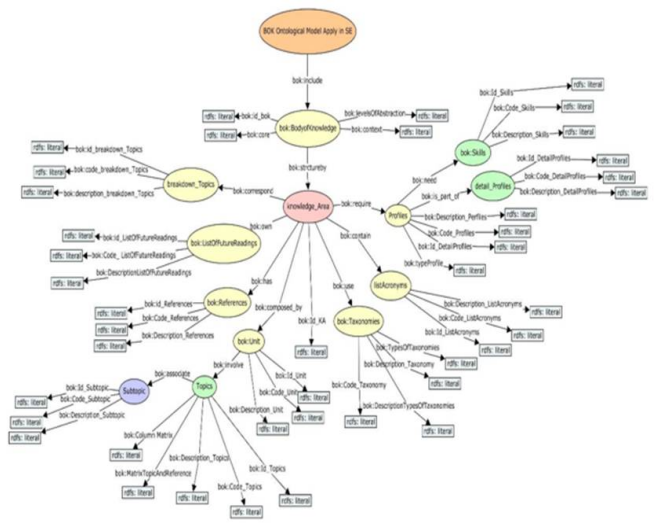

Figure 3: Initial Ontology in SEC [6]
Another way to describe the knowledge in the engineering context is focused in capacities; in [18], consider 30 capacities to develop the correct way a BOK in SEC.

One of the learning lesson in the process of describing a knowledge were establishment of skills, and attitudes of stakeholder and those of the organizations [19]. The competencies involve the mobilization of practical, and cognitive skills, creative skills, and other resources like attitudes, motivation, and values to apply in the BOK domain, and context.

The knowledge description for Bodies of Knowledges allow a validated classification of the bounds of the discipline and topical access that will support the discipline; it is subdivided into a set of KA allowing readers to find their way quickly to subjects of interest and create its professional profiles.

Our model is capable of representing rich semantic knowledge, including both, skills and concepts, integrating them through a coherent network of associations, in terms of context specification, based on a constructivist approach.

\section{CONCLUSION}

A BOK generally uses a tree structure to represent knowledge, and a certain limit is set to its height to help its understand ability and readability. In the same context the main objective of a BOK is to provide classification of knowledge, and its detailed explanation.

The definition of BOK in the context of software engineering is important to respond the training needs of future professional to they acquire the competencies in the social, business, educational and industrial.

The BOK provides the basis for curriculum development and maintenance and supports professional development and any current and future certification schemes. Lastly, it promotes integration and connections with related disciplines.

The BOK is the sum total of our human understanding of the world around us. Studies in the area of strength and conditioning make up one of the many fields of knowledge, and strength and conditioning professionals must understand how our understanding is created to successfully use it to optimize their professional practices, approaches, and exercise prescriptions.

The SWEBOK is an all-inclusive term that describes the sum of knowledge within the profession of software engineering. How- ever, the Guide to the SWEBOK seeks to identify and describe that subset of the body of knowledge that is generally accepted or, in other words, the core body of knowledge.

A SWEBOK can fulfill to stakeholder's role in supporting education, certification, professional stature, professional development, and organizational improvement.

Standard taxonomies, such as the SE2004, and SWEBOK, can provide useful input regarding the knowledge content of the 
course and how the course may be best integrated into the computer engineering curriculum.

\section{FUTURE WORK}

This paper is a first effort towards building a model to support knowledge description for SWEBOK. The next step will be to define a model for BOK based on the conclusions obtained from the current study.

\section{ACKNOWLEDGE}

The authors express their gratitude to the Waseda University, Universidad Internacional del Ecuador (UIDE), Universidad Tecnica Particular de Loja, Technical University of Madrid (UPM), in particular to the Doctoral Program of Science and Technology of Computing for Smart Cites.

\section{REFERENCES}

[1] B. Penzenstadler, D. Mendez Fernandez, D. Richardson, D. Callele, and K. Wnuk, "The requirements engineering body of knowledge (rebok)," in Requirements Engineering Conference (RE), 2013 21st IEEE International, pp. 377-379, July 2013.

[2] K. Taguchi, H. Nishihara, T. Aoki, F. Kumeno, K. Hayamizu, and K. Shinozaki, "Building a body of knowledge on model checking for software development," in Computer Software and Applications Conference (COMPSAC), 2013 IEEE 37th Annual, pp. 784-789, July 2013.

[3] Licensure and Q. for Practice Committee of the National Society of Professional Engineers, "Engineering body of knowledge," in National Society of Professional Engineers (NSPE), p. 67 pages, 2013.

[4] C. Smith and D. J. Brooks, Security Science: The Theory and Practice of Security. Newton, MA, USA: Butterworth-Heinemann, 2013.

[5] D. A. Mundie and R. Ruefle, "Building an incident management body of knowledge," in Proceedings of the 2012 Seventh International Conference on Availability, Reliability and Security, ARES '12, (Washington, DC, USA), pp. 507-513, IEEE Computer Society, 2012.

[6] Quezada, P., Garbajosa, J., Enciso, L. Use of standard and model based on BOK to evaluate professional and occupational profiles (2016) Advances in Intelligent Systems and Computing, 444, pp. 287-296. DOI: 10.1007/978-3319-31232-3 2

[7] P. Bourque, L. Buglione, A. Abran, and A. April, "Bloom's taxonomy levels for three software engineer profiles," in Software Technology and Engineering Practice, 2003. Eleventh Annual International Workshop on, pp. 123-129, Sept 2003.

[8] "Using ontologies for knowledge management: An information systems perspective," An information systems perspective, " Proc. ASIS Annual Mtg, vol. 3, pp. 482-496, 1999.

[9] M. Azuma, F. Coallier, and J. Garbajosa, "How to apply the bloom taxonomy to software engineering," in Software Technology and Engineering Practice, 2003. Eleventh Annual International Workshop on, pp. 117-122, Sept 2003.

[10] A. Hunter and W. Liu, "A survey of formalisms for representing and reasoning with scientific knowledge," Knowledge Eng. Review, vol. 25, no. 2, pp. 199-222, 2010.

[11] K. Peffers, T. Tuunanen, M. Rothenberger, and S. Chatterjee, "A design science research methodology for information systems research," J. Manage. Inf. Syst., vol. 24, pp. 45-77, Dec. 2007.

[12] A. Carusi and A. de Waard, "Changing modes of scientific discourse analysis, changing perceptions of science," in E-Science Workshops, 2009 5th IEEE International Conference on, pp. 194-195, Dec 2009.

[13] C. Ramirez and B. Valdes, "A general knowledge representation model for the acquisition of skills and concepts," in Cognitive Informatics, 2009. ICCI '09. 8th IEEE International Conference on, pp. 412-417, June 2009.

[14] P. A. Quezada-Sarmiento, L. E. Enciso-Quispe, J. Garbajosa and H. Washizaki, "Curricular design based in bodies of knowledge: Engineering education for the innovation and the industry," 2016 SAI Computing $\begin{array}{llll}\text { Conference (SAI), } & \text { London, 2016, } & \text { p43-849. }\end{array}$ 10.1109/SAI.2016.7556077 URL: http://ieeexplore.ieee.org/stamp/stamp.jsp?tp=\&arnumber=7556077\&is number $=7555953$

[15] F. Alvarez, A. Vaquero, and F. Saenz, "Some software engineering and knowledge representation principles for the design and construction of ontology-based linguistic resources," in Intelligent Systems Design and Applications, 2008. ISDA '08. Eighth International Conference on, vol. 2, pp. 110-115, Nov 2008.

[16] P. Wongthongtham, N. Kasisopha, E. Chang, and T. Dillon, "A software engineering ontology as software engineering knowledge representa- tion," in Convergence and Hybrid Information Technology, 2008. ICCIT '08. Third International Conference on, vol. 2, pp. 668-675, Nov 2008.

[17] W. Jianping, "A novel software engineering knowledge representation method for multi-site software development," in Software Engineering and Service Science (ICSESS), 2012 IEEE 3rd International Conference on, pp. 523-526, June 2012.

[18] Licensure and Q. for Practice Committee of the National Society of Professional Engineers, "Engineering body of knowledge," in National Society of Professional Engineers (NSPE), p. 67 pages, 2013.

[19] P. A. Quezada-Sarmiento, L. Enciso and J. Garbajosa, "Use of body knowledge and cloud computing tools to develop software projects based in innovation," 2016 IEEE Global Engineering Education Conference (EDUCON), Abu Dhabi, 2016, pp. 267-272. doi:10.1109/EDUCON.2016.7474564

URL: http://ieeexplore.ieee.org/stamp/stamp.jsp?tp=\&arnumber=7474564\&is number $=7474513$ 